\title{
The Role of Population Size in Rate of Evolution in Genetic Programming
}

\author{
Ting $\mathrm{Hu}$ and Wolfgang Banzhaf \\ Department of Computer Science, Memorial University, St. John's, Canada \\ \{tingh, banzhaf\}@cs.mun.ca
}

\begin{abstract}
Population size is a critical parameter that affects the performance of an Evolutionary Computation model. A variable population size scheme is considered potentially beneficial to improve the quality of solutions and to accelerate fitness progression. In this contribution, we discuss the relationship between population size and the rate of evolution in Genetic Programming. We distinguish between the rate of fitness progression and the rate of genetic substitutions, which capture two different aspects of a GP evolutionary process. We suggest a new indicator for population size adjustment during an evolutionary process by measuring the rate of genetic substitutions. This provides a separate feedback channel for evolutionary process control, derived from concepts of population genetics. We observe that such a strategy can stabilize the rate of genetic substitutions and effectively accelerate fitness progression. A test with the Mackey-Glass time series prediction verifies our observations.
\end{abstract}

\section{Introduction}

The search process in Evolutionary Computation (EC) systems is a simultaneous process of exploration in parallel and exploitation in depth. Population size is a key factor to maintain population diversity in this process, and thus critical for the performance of an EC method. Recently, population size control has attracted increasing interests in the literature 13. Population size control is non-trivial and challenging because it is often problem-specific and the interaction among various EC parameters is not completely clear yet. In general, the literature on population size control has two main foci: i) initializing a proper population size a priori and ii) adjusting population size during evolution. In this article, we focus on the latter. Population size adjustment is motivated by the observation that the required population size changes during different stages of evolution [2]. Such an adjustment is usually directed by a feedback loop. This feedback has been implemented through the controlled persistence of individuals or through the measurement of fitness progression, both of which are able to reflect the process of evolution to some extent. In Biology, particularly in the study on population genetics, population size has been intensely studied regarding its role in the rate of evolution 1516. It is generally accepted that the effect of population size on evolution acceleration is conditioned on the nature of selection at a particular moment rather than on a monotonic relationship. Typically,

L. Vanneschi et al. (Eds.): EuroGP 2009, LNCS 5481, pp. 85-96, 2009.

(C) Springer-Verlag Berlin Heidelberg 2009 
under positive selection, i.e., selection mostly accepting adaptive phenotypes, a large population is favorable for rapid evolution. In contrast, under negative selection, i.e., selection mostly eliminating deleterious phenotypes, a small population evolves faster. These two selection conditions can be reflected by the rate of genetic substitutions. Although this perspective is still under debate in the biological community, it is intriguing to study this relationship in EC systems. Thus, we investigate the interplay between population size and rate of evolution in a GP model and see how this originally biological notion translates to artificial systems.

\section{Background and Motivation}

In this section, we first briefly review studies on population size control in EC. Then, we discuss the relation between population size and the rate of evolution from both an EC and biological point of view.

\subsection{Population Size Control}

Research on population size control in EC originated from Genetic Algorithms (GAs). A number of theoretical contributions on analyzing population size initialization have been published based on Goldberg's seminal "components decomposition approach" and the notion of building blocks 89. The essence of these works is that population size should be initialized according to the "complexity" of a specific problem. That is, for a more difficult problem, more diversity of a population is required, and thus a larger population size should be initialized.

Recently, it was realized that even for a given problem instance the required population size can vary during the process of evolution. Therefore, besides a good initial population size, some empirical methods on adjusting population size dynamically have been proposed. Arabas et al. 1] propose the Genetic Algorithm with Variable Population Size (GAVaPS) by regulating the age and lifetime of each individual. Population size fluctuates as a result of removing over-aged individuals and reproducing new ones. Back et al. [2] extend this lifetime notion in their Adaptive Population size Genetic Algorithm (APGA) to steady-state GAs. Fernandes and Rosa [5] propose the Self-Regulated Population size Evolutionary Algorithm (SRP-EA) to enhance APGA using a diversity-driven reproduction process. Alternatively, Harik and Lobo [10] introduce parameter-less GA, where several populations with different sizes evolve in parallel, starting with small population sizes. By inspecting the average fitness of these populations, less fit undersized populations are replaced by larger ones. Eiben et al. 4] suggest to use the pace of fitness improvements as the signal to control population size in Population Resizing on Fitness Improvement GA (PRoFIGA).

In GP, determination of an ideal population size is of even greater significance. As with the GA, population size in GP is relevant to its capabilities in finding the target and to its computational efficiency. In particular, it is related 
to the phenomenon of bloat. Poli et al. [17] establish that smaller populations bloat at a slower rate than larger ones. Downing [3] investigates population size in relation to evolvability in GP. Thus, adjusting population size dynamically benefits GP in various ways. A theoretical analysis on population size in GP based on building blocks is conducted by Sastry et al. 18. The empirical population size adjustment schemes for GAs can also be applied to GP. Moreover, some GP-specific techniques have been employed as well. For example, Wedge and Kell [20] propose the Genotype-Fitness Correlation as a landscape metric to predict ideal population sizes in different systems. Tomassini et al. [19] design a dynamic population size GP using fitness progression as signal to delete over-sized and worse-fit individuals or to insert mutated best-fit individuals with certain criteria.

\subsection{Population Size and Rate of Evolution}

The term rate of evolution is understood somewhat differently in EC and in Biology. The goal of evolution is much more explicit in computational systems than in nature. It is to find the fittest solution to a given problem. Therefore, the rate of evolution in EC usually refers to how fast an EC population improves its fitness value, i.e., the rate of fitness progression. In this sense, as long as an EC population is able to find solutions, a small size is favored because of a small overhead. Thus, computer scientists have been seeking intelligent population size control schemes to strike a balance between exploration and exploitation during the search process.

In Biology, there is no explicit fitness function to measure, and the rate of evolution comes in a few different flavors, depending on the objects being examined, such as gene sequences, proteins, etc. Because of the infeasibility of defining fitness explicitly in natural systems, fitness is usually reflected by the likelihood that a relevant genetic change is selected. For instance, in molecular biology, the rate of evolution is usually measured by the rate that mutants are accepted and replace former alleles in genetic sequences. Biologists refer to this rate of evolution as rate of genetic substitutions. Here, we distinguish the rate of fitness progression and the rate of genetic substitutions to acknowledge the two aspects of rate of evolution. Fitness progression focuses on attaining the goal of the search, while rate of genetic substitutions concentrates on the dynamics of evolution and provides a different tool to study evolutionary processes.

Population geneticists have been identifying the effect of population size on the rate of molecular evolution, i.e., the rate of genetic substitutions. The Nearly Neutral Theory of molecular evolution by Ohta [1516] is regarded as one of the most important principles for modern molecular evolution research. This theory defines both slightly deleterious and slightly advantageous mutations as nearly neutral mutations. It extends an earlier insight of Fisher [6] that the probability of a mutant being selected will be low if the outcome of this mutation on phenotypes is far-reaching. The theory predicts that there are a substantial number of nearly neutral mutations in molecular evolution. These nearly neutral mutations would be able to generate adaptation at a later time under certain genetic or 
environmental changes. Thus, they play an important role for providing variation potential. In this theory, population size can influence the rate of molecular evolution by its effects on the chance of accepting a nearly neutral genetic change through statistical laws. That is, the chance of a random mutant being favored by selection is slimmer within a larger population. When the majority of mutations are deleterious, a smaller population can evolve faster because more nearly neutral changes are introduced into the population. In contrast, when mutations are mostly advantageous, evolution is faster in a larger population. When most mutations are neutral, the rate of evolution is nearly independent of the population size.

These predictions have been extensively tested and discussed in the biological community. Below are some examples where both increasing and decreasing population size may accelerate evolution, depending on the link to environments. Gillespie [7] examines the relation between population size and the rate of genetic substitutions via computer simulation of several well-known biological models. While verifying such a relation, he suggests the relation can sometimes be blurred by the extreme complexity of natural systems. With population size fluctuation being one of these complicating factors, he further emphasizes the necessity of studying such fluctuation in population genetics. Woolfit and Bromham 21] compare genetic sequences between island endemic species and closely related mainland species. This is an example where decreasing population size can accelerate evolution. In a study on the recent rapid molecular evolution in human genomes, Hawks et al. 11] hypothesize that the current dramatically growing human population may be the major driving force of new adaptive evolution. They indicate that a growing population size can provide the potential for rapid adaptive innovations if a population is highly adaptive to the current environment.

\section{Adjusting Population Size during Evolution in GP}

We propose to apply the ideas from population genetics to a GP system. It is generally assumed that the fitness of new offspring generated by mutation or crossover in each generation approximately follows a Gaussian distribution. According to the Central Limit Theorem, the average fitness among a larger population has a smaller variance [15] (Fig. 1). We use the selection favoring degree $S_{f}$, similar to the selection coefficient in Biology, to denote the degree of new offspring being favored by selection. A positive value of $S_{f}$ of an offspring implies that it is likely to be accepted, and a negative value of $S_{f}$ means that it will most likely be rejected by selection. Further, if the majority of offspring have positive $S_{f}$, selection is referred to as positive (Fig. 1 left). In contrast, the selection is negative when $S_{f}<0$ for most offspring (Fig. 1 right). From this figure, we observe that, under positive selection, increasing population size can accelerate the rate of genetic substitutions (left), while decreasing population size can allow more genetic substitutions under negative selection (right).

Selection acting positively or negatively may vary during different stages of an evolutionary process in GP, and the rate of genetic substitutions reflects this 

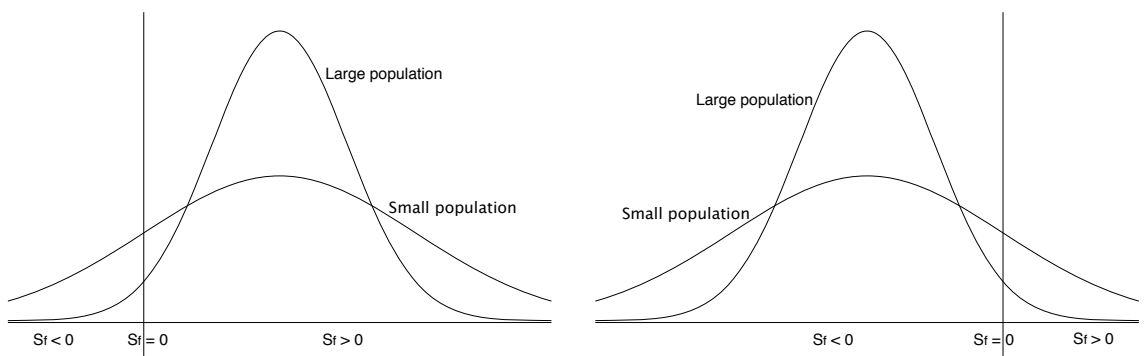

Fig. 1. Selection effects. Positive selection (left) and negative selection (right).

varying selection pressure. Therefore, adjusting population size according to the rate of genetic substitutions is expected to compensate the selection pressure. Thus, evolution can be guided away from stagnation and, further, can achieve better fitness progression. A measurement for the rate of genetic substitutions is reviewed briefly next, followed by our proposed population size adjustment approach.

\subsection{Measuring the Rate of Genetic Substitutions}

The nonsynonymous to synonymous substitution ratio $k_{\mathrm{a}} / k_{\mathrm{s}}$ is a widely accepted measurement of the rate of genetic substitutions in molecular biology [23. This metric has been applied by us 12 to measure the rate of genetic substitutions in GP. Not all genetic changes are effective in GP. Genetic changes that modify the encoded function can be regarded nonsynonymous, while others are regarded as synonymous changes. Specifically, in each generation the population is formed by surviving individuals under genetic variations and selection. Then, $k_{\mathrm{a}}$ measures the rate of nonsynonymous genetic substitutions of the process, and $k_{\mathrm{s}}$ measures the rate of neutral changes accepted. The rates $k_{\mathrm{a}}$ and $k_{\mathrm{s}}$ are obtained by counting the number of nonsynonymous (synonymous, resp.) genetic substitutions normalized by the total nonsynonymous (synonymous, resp.) sensitivities of individuals to genetic operations in each generation. Therefore, $k_{\mathrm{a}}$ measures the adaptive evolutionary "distance" (adaptive substitution rate) and $k_{\mathrm{s}}$ practically provides the background "clock ticks" (neutral substitution rate). In Biology, the ratio $k_{\mathrm{a}} / k_{\mathrm{s}}$ is regarded as a measure for the rate of genetic substitutions. On one hand, $k_{\mathrm{a}} / k_{\mathrm{s}}>1$ implies that most genetic changes are adaptive and favored by selection. On the other hand, when $k_{\mathrm{a}} / k_{\mathrm{s}}<1$, the majority of genetic changes are regarded deleterious and are diminished by selection.

Here, we slightly revise this $k_{\mathrm{a}} / k_{\mathrm{s}}$ ratio to measure the rate of genetic substitutions. From one generation to the next, $N_{\mathrm{a}}$ denotes the number of all nonsynonymous genetic changes (i.e., attempted changes) and $M_{\mathrm{a}}$ counts the number of nonsynonymous genetic substitutions (i.e., accepted changes). A sampled semantic test set different from the training set is fed to an individual before and after a genetic change to test whether this change is nonsynonymous or synonymous. For instance, if a parent and its offspring have the same output for all sampled 
semantic test cases, the genetic change generating this offspring from the parent is regarded as synonymous. Otherwise, the change is considered nonsynonymous. Thus, $k_{\mathrm{a}}=M_{\mathrm{a}} / N_{\mathrm{a}}$ measures the rate of nonsynonymous genetic substitutions. The synonymous substitution rate $k_{\mathrm{s}}$ can be defined similarly by dividing the number of synonymous genetic substitutions $M_{\mathrm{s}}$ by the number of attempted synonymous genetic changes $N_{\mathrm{s}}$. The ratio $k_{\mathrm{a}} / k_{\mathrm{s}}$ measures the rate of adaptive genetic substitutions relatively to a background silent genetic substitution rate. The case $k_{\mathrm{a}} / k_{\mathrm{s}}=1$ corresponds to the situation where nonsynonymous genetic changes are selected at the same rate as neutral changes. When $k_{\mathrm{a}} / k_{\mathrm{s}}>1$, selection is positive because a larger portion of nonsynonymous changes are favored by selection. In contrast, negative selection is reflected by the case $k_{\mathrm{a}} / k_{\mathrm{s}}<1$.

\subsection{Adaptive Population Size Approach}

Next, an adaptive population size scheme is proposed using the $k_{\mathrm{a}} / k_{\mathrm{s}}$ ratio defined above. We adopt truncation selection such that population size adjustment can be achieved easily without duplication or generating random individuals. Typically, at generation $t$, the current population produces an offspring population of the same size via genetic variations including crossover and mutation. Parents and offspring will compete through tournament selection to yield the next generation, and the population size $P_{\text {size }}(t+1)$ will be adjusted according to the currently observed rate of genetic substitutions $\left(k_{\mathrm{a}} / k_{\mathrm{s}}\right)(t)$. Thus, the adaptive population size is regulated in each generation in an attempt to maintain a stable rate of genetic substitutions as follows:

- If $\left(k_{\mathrm{a}} / k_{\mathrm{s}}\right)(t)>1$ (positive selection), we increase the population size proportional to the changes of the rate of genetic substitutions such that,

$$
P_{\text {size }}(t+1)=P_{\text {size }}(t) \times\left(1+\left|\left(k_{\mathrm{a}} / k_{\mathrm{s}}\right)(t)-\left(k_{\mathrm{a}} / k_{\mathrm{s}}\right)(t-1)\right|\right) .
$$

- If $\left(k_{\mathrm{a}} / k_{\mathrm{s}}\right)(t)=1$ (neutral selection), we keep the same population size that,

$$
P_{\text {size }}(t+1)=P_{\text {size }}(t) \text {. }
$$

- If $\left(k_{\mathrm{a}} / k_{\mathrm{s}}\right)(t)<1$ (negative selection), when $\left(k_{\mathrm{a}} / k_{\mathrm{s}}\right)(t)$ is increasing, we increase the population size to suppress further deleterious genetic substitutions, and when $\left(k_{\mathrm{a}} / k_{\mathrm{s}}\right)(t)$ is decreasing, we decrease the population size to encourage more genetic substitutions. That is,

$$
P_{\text {size }}(t+1)=P_{\text {size }}(t) \times\left(1+\left(\left(k_{\mathrm{a}} / k_{\mathrm{s}}\right)(t)-\left(k_{\mathrm{a}} / k_{\mathrm{s}}\right)(t-1)\right)\right) .
$$

Note that we do not limit the population size by an upper bound. However, a lower bound of population size will be established in applications.

\section{Experiments}

We expect that dynamic adjustment of population size according to the measured $k_{\mathrm{a}} / k_{\mathrm{s}}$ ratio can maintain a fairly stable rate of genetic substitutions. Since 


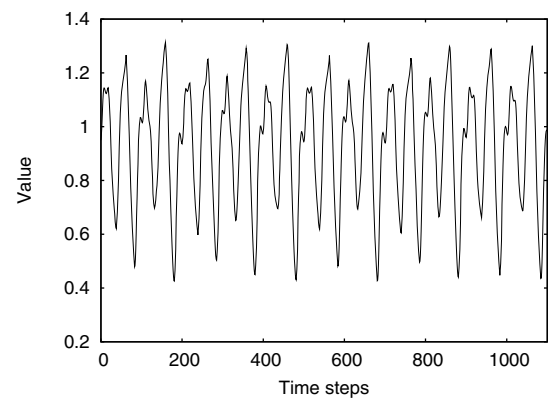

Fig. 2. Mackey-Glass time series

evolution is better guided this way, the performance of a GP system in fitness progression should improve as well. This is verified through simulations and comparisons to fixed-size populations. A tree structure is adopted to encode GP individuals here. The test benchmark will be introduced next, followed by our discussion of experimental results.

\subsection{Test Suite}

We use the Mackey-Glass chaotic time series prediction as our benchmark problem. Mackey-Glass time series prediction is a difficult modeling problem in machine learning and in GP 14. It predicts future values of a time series based on history. GP is trained using these historical data. The series is generated using the following recursive function [22,

$$
x_{t+1}=x_{t}-b \times x_{t}+\frac{a \times x_{t-\tau}}{1+\left(x_{t-\tau}\right)^{10}},
$$

where $x_{0}=1$, and the parameters are set to

$$
a=0.2, b=0.1, \tau=17
$$

Fig. 2 depicts a plot of this function. We use the first 1,001 points as the training set. This problem is considered a difficult one because it does not have a closed-form solution. Thus, it will take GP a long time to converge.

Empirically, a population size between 500 and 1,000 is suitable for this type of problem. Here we conduct experiments in three scenarios. Two of the scenarios have fixed-size populations of 500 and 1,000, the third has an adaptive population size (APS) using our dynamic adjustment approach. It starts with an initial population size of 1,000 and a lower limit 300. The GP configuration is as shown in Table 1. Note that we adopt the number of function evaluations as a control metric although it operates in a generational mode. This allows a fair comparison among different scenarios. 
Table 1. GP Configuration

\begin{tabular}{l||l}
\hline Population size & $500 / 1,000 /$ APS(Adaptive Population Size) \\
\hline Tree initialization & Ramped-Half-and-Half with limit 6 \\
\hline Function set &,,$+- \times$, and protective $/$ \\
\hline Terminal variable set & $\begin{array}{l}x_{1}, x_{2}, \ldots, \text { and } x_{17}, \text { variable } x_{i} \text { denotes the } \\
\text { previous point } i \text { time steps ago }\end{array}$ \\
\hline Terminal constant set & $\begin{array}{l}\text { Random ephemeral numbers equally dis- } \\
\text { tributed in }[-1,1] \text { with granularity } 0.01\end{array}$ \\
\hline Crossover rate & 0.9 \\
\hline Mutation rate & 0.1 \\
\hline Maximum mutation subtree depth & 4 \\
\hline Crossover and mutation method & Subtree replacement \\
\hline Maximum tree depth & 100 \\
\hline Training set & Points from 0 to 1,000 time steps \\
\hline Fitness function & Root Mean Square $(\mathrm{RMS})$ error \\
\hline Selection & Tournament with size 4 \\
\hline Sampled sematic test set & 20 cases such that $x_{i}^{j}=(i+j-2) \times 0.04$ \\
\hline Maximum number of evaluations & $(1 \leq i \leq 17,1 \leq j \leq 20), 0 \leq x_{i}^{j} \leq 1.4$ \\
\hline
\end{tabular}

\subsection{Results and Discussion}

We have run GP 200 times for each scenario. Before we present statistical results, we look into the details of a "typical" execution of the APS scenario (Fig. 3). This particular population evolves for 147 generations before it reaches the 100,000 function evaluation number limit. In the figure, we plot (a) best fitness, (b) $k_{\mathrm{a}} / k_{\mathrm{s}}$ ratio, (c) average tree size, and (d) population size over generations. We observe that the $k_{\mathrm{a}} / k_{\mathrm{s}}$ ratio stays well under 1 , which implies that selection is negative over time. This concurs with the general understanding that random genetic changes are mostly deleterious and with the property of the $k_{\mathrm{a}} / k_{\mathrm{s}}$ ratio in Biology 23. The population size drops from an initial 1,000 to approximately 700 after 20 generations, and stabilizes at 650 700 afterwards. Also notice that, as evolution progresses, the best fitness improves but at a slower rate and average tree size increases, which is expected for tree GP. Normally, bloat would slow down the rate of genetic substitutions due to the introduction of redundant substructures. However, this is successfully alleviated by adjusting the population size to stimulate evolution so that there is a steady $k_{\mathrm{a}} / k_{\mathrm{s}}$ ratio. This is verified by our next close study of the interaction between the $k_{\mathrm{a}} / k_{\mathrm{s}}$ ratio and population size.

In Fig. 4, we depict the response of the $k_{\mathrm{a}} / k_{\mathrm{s}}$ ratio change to population size adjustment, derived from the data recorded from 200 runs of the APS scheme. Using the recorded population size and the $k_{\mathrm{a}} / k_{\mathrm{S}}$ ratio of each run, we quantify the correlation between the way they change over generations using a sequence of 1's and -1 's. For a generation compared to the previous, if both population size and the $k_{\mathrm{a}} / k_{\mathrm{s}}$ ratio increase, or if both population size and the $k_{\mathrm{a}} / k_{\mathrm{s}}$ ratio 
(a) Fitness progression

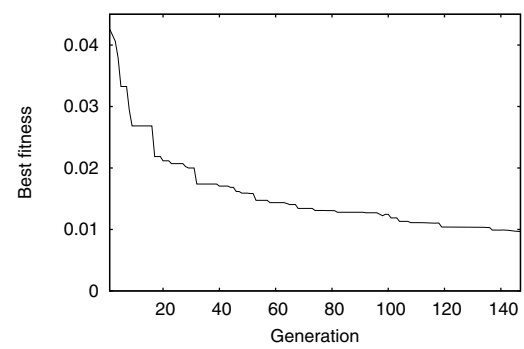

(c) Average tree size

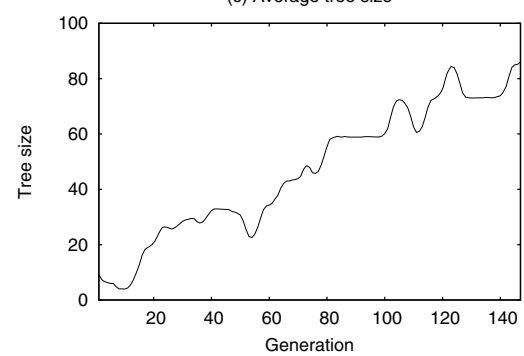

(b) Rate of genetic substitutions

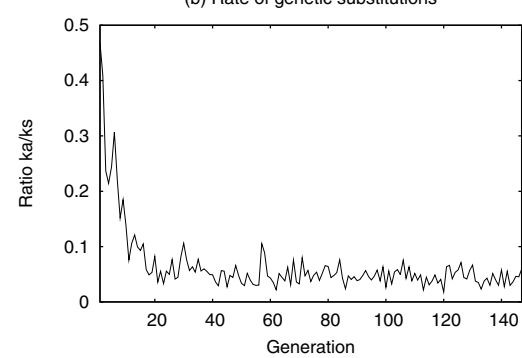

(d) Population size

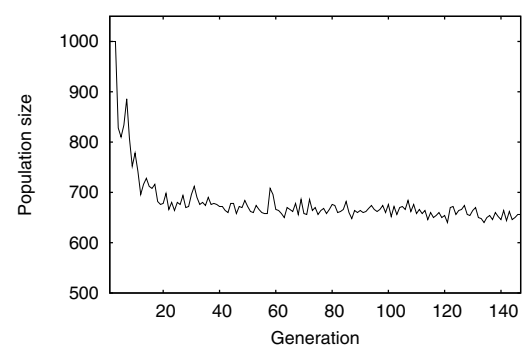

Fig. 3. An example run with adaptive population size according to the rate of genetic substitutions $k_{\mathrm{a}} / k_{\mathrm{s}}$ ratio

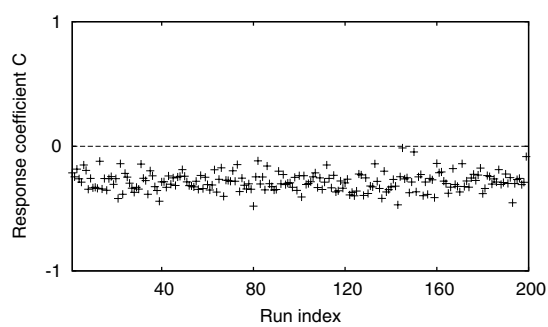

Fig. 4. Correlations between population size and $k_{\mathrm{a}} / k_{\mathrm{s}}$ ratio

decrease, we have a 1 . Otherwise, we have a -1 . Therefore, the number of 1 's in the produced sequence records the number of occurrences where the change of the $k_{\mathrm{a}} / k_{\mathrm{s}}$ ratio positively correlates to that of the population size; while -1 's indicate negative correlation. We define the response coefficient $C$ as $C=(2 n-l) / l$, where $n$ is the number of $1 \mathrm{~s}$ in the sequence and $l$ is the sequence length. Thus, if a run has $C=0$, its $k_{\mathrm{a}} / k_{\mathrm{s}}$ ratio is independent of the change of population size. Alternatively, a positive value of $C$ indicates a positive correlation between the changes of the $k_{\mathrm{a}} / k_{\mathrm{s}}$ ratio and that of the population size. On the other hand, a negative value of $C$ suggests a negative correlation. The figure presents the coefficients for all the 200 simulation runs. Clearly, they are all well below the level of 0 . This is indeed our intention of dynamically adjusting population size to stabilize the rate of genetic substitution as stated in Section 3.2 
Table 2. Mean Best Fitness $\left(\times 10^{-3}\right)$ Comparison

|Mean Best Fitness|Standard Deviation|Median|95\% Confidence Interval

\begin{tabular}{|c|c|c|c|c|}
\hline Psize 500 & 13.980 & 6.941 & 12.36 & {$[13.153,14.806]$} \\
\hline Psize 1,000 & 12.902 & 5.406 & 12.98 & {$[12.195,13.610]$} \\
\hline APS & 12.053 & 4.750 & 11.56 & {$[11.226,12.888]$} \\
\hline
\end{tabular}

(a) The percentage of runs to a fitness

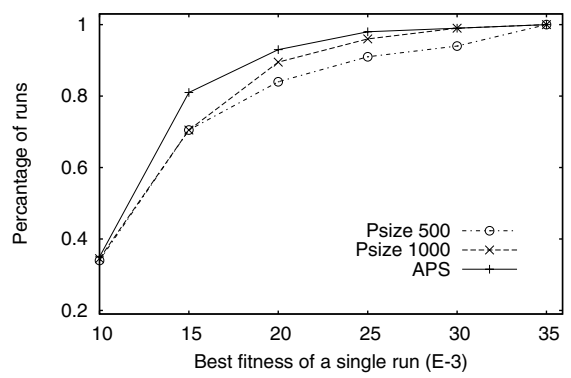

(b) The average number of evaluations to a fitness

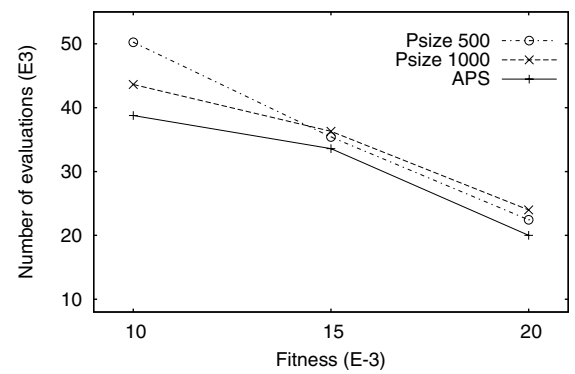

Fig. 5. Fitness progression with fixed population sizes (Psize 500, Psize 1000) and adaptive population size (APS)

Our next observation is that fitness progression can also be accelerated by our population size adjustment scheme. Here, we adopt the three most commonly used metrics to measure the performance of an EC model. They are mean best fitness, success rate, and average number of evaluations to a solution. Table 2 presents the mean best fitness and standard deviation over 200 runs for the three scenarios of fixed population size 500, 1,000, and APS. Clearly, APS achieves an even better fitness than maintaining 1,000 individuals but its population size is mostly between 500 and 1,000. The cumulative success rates of these three groups are depicted in Fig. [5(a). We focus on the best fitness of a run once it terminates. The figure plots the percentage of the total 200 runs of each scenario that yield a better fitness than a given threshold between 0.01 and 0.035. Apparently, APS has the highest percentage for all of the cases, which indicates its superiority over fixed population size strategies. Fig. [5) (b) reveals a dual measure for the three scenarios. In this chart, we compare the average number of evaluations needed for a simulation run to achieve fitness levels of $0.01,0.15$, and 0.02 . For a given fitness level, APS always incurs less computation overhead, and this difference becomes greater as the fitness requirement gets higher.

\section{Conclusion and Future Work}

In this article, we investigated the role of population size for rate of evolution in a GP system. We distinguished between rate of genetic substitutions and rate of fitness progression, which describe two aspects of an evolutionary process. The 
measurement of rate of genetic substitutions was revised and adopted. We transferred an idea from population genetics that population size has varying effects on rate of genetic substitutions under differing selection regimes. We proposed and tested that dynamically adjusting population size can effectively stabilize the rate of genetic substitutions even during late stages of an evolutionary process. It has been further verified that this strategy can also successfully accelerate the rate of fitness progression.

Our observations suggest some future research. First, we would like to implement this idea in GP with different representation structures, e.g., Linear GP and Graph GP, to see if similar conclusions hold in these GP systems. Applications to other branches of Evolutionary Computation, e.g., GAs, are also expected. Second, we will investigate this adaptive population size scheme in changing environments, since dynamic environments may affect the regime of selection more dramatically than static environments. Third, we propose to apply our method to other benchmarks in order to test how it fares compared to other well-known adaptive population size strategies.

\section{Acknowledgements}

We thank NSERC for support under Discovery Grant RGPIN 283304-07.

\section{References}

1. Arabas, J., Michalewicz, Z., Mulawka, J.: GAVaPS - a genetic algorithm with varying population size. In: Proceedings of IEEE Congress on Evolutionary Computation (CEC 1994), pp. 73-78. IEEE Press, Los Alamitos (1994)

2. Back, T., Eiben, A.E., van der Vaart, N.A.L.: An empirical study on GAs "without parameters". In: Deb, K., Rudolph, G., Lutton, E., Merelo, J.J., Schoenauer, M., Schwefel, H.-P., Yao, X. (eds.) PPSN 2000. LNCS, vol. 1917, pp. 315-324. Springer, Heidelberg (2000)

3. Downing, R.M.: On population size and neutrality: Facilitating the evolution of evolvability. In: Ebner, M., O’Neill, M., Ekárt, A., Vanneschi, L., Esparcia-Alcázar, A.I. (eds.) EuroGP 2007. LNCS, vol. 4445, pp. 181-192. Springer, Heidelberg (2007)

4. Eiben, A.E., Marchiori, E., Valkó, V.A.: Evolutionary algorithms with on-the-fly population size adjustment. In: Yao, X., Burke, E.K., Lozano, J.A., Smith, J., Merelo-Guervós, J.J., Bullinaria, J.A., Rowe, J.E., Tiňo, P., Kabán, A., Schwefel, H.-P. (eds.) PPSN 2004. LNCS, vol. 3242, pp. 41-50. Springer, Heidelberg (2004)

5. Fernandes, C., Rosa, A.: Self-regulated population size in evolutionary algorithms. In: Runarsson, T.P., Beyer, H.-G., Burke, E.K., Merelo-Guervós, J.J., Whitley, L.D., Yao, X. (eds.) PPSN 2006. LNCS, vol. 4193, pp. 920-929. Springer, Heidelberg (2006)

6. Fisher, R.A.: Genetical Theory of Natural Selection. Clarendon, Oxford (1930)

7. Gillespie, J.H.: The role of population size in molecular evolution. Theoretical Population Biology 55(2), 145-156 (1999)

8. Goldberg, D.E.: Sizing populations for serial and parallel genetic algorithms. In: Proceedings of the 3rd International Conference on Genetic Algorithms, pp. 70-79. Morgan Kaufmann, San Francisco (1989) 
9. Goldberg, D.E., Deb, K., Clark, J.H.: Genetic algorithms, noise, and the sizing of populations. Complex Systems 6(4), 333-362 (1992)

10. Harik, G.R., Lobo, F.G.: A parameter-less genetic algorithm. In: Proceedings of the Genetic and Evolutionary Computation Conference (GECCO 1999), pp. 258-267. Morgan Kaufmann, San Francisco (1999)

11. Hawks, J., Wang, E.T., Cochran, G.M., Harpending, H.C., Moyzis, R.K.: Recent acceleration of human adaptive evolution. Proceedings of the National Academy of Sciences 104(52), 20753-20758 (2007)

12. $\mathrm{Hu}, \mathrm{T}$., Banzhaf, W.: Nonsynonymous to synonymous substitution ratio $k_{\mathrm{a}} / k_{\mathrm{s}}$ : Measurement for rate of evolution in evolutionary computation. In: Rudolph, G., Jansen, T., Lucas, S., Poloni, C., Beume, N. (eds.) PPSN 2008. LNCS, vol. 5199, pp. 448-457. Springer, Heidelberg (2008)

13. Lobo, F.G., Lima, C.F.: A review of adaptive population sizing schemes in genetic algorithms. In: Proceedings of the 2005 workshops on Genetic and Evolutionary Computation (GECCO 2005), pp. 228-234. ACM, New York (2005)

14. Oakley, H.: Two scientific applications of genetic programming: stack filters and non-linear equation fitting to chaotic data. In: Kinnear, K.L., Kinnear Jr., K.E. (eds.) Advances in Genetic Programming, pp. 369-389. MIT Press, Cambridge (1994)

15. Ohta, T.: Population size and rate of evolution. Journal of Molecular Evolution 1(4), 305-314 (1972)

16. Ohta, T.: The nearly neutral theory of molecular evolution. Annual Reviews in Ecology and Systematics 23(1), 263-286 (1992)

17. Poli, R., McPhee, N.F., Vanneschi, L.: The impact of population size on code growth in GP: analysis and empirical validation. In: Proceedings of the 10th Annual Conference on Genetic and Evolutionary Computation (GECCO 2008), pp. 12751282. ACM, New York (2008)

18. Sastry, K., O'Reilly, U.-M., Goldberg, D.E.: Population sizing for Genetic Programming based upon decision making. In: O'Reilly, U.-M., Yu, T., Riolo, R., Worzel, B. (eds.) Genetic Programming Theory and Practice II, pp. 49-66. Kluwer Academic Publishers, Dordrecht (2004)

19. Tomassini, M., Vanneschi, L., Cuendet, J.: A new technique for dynamic size populations in genetic programming. In: Proceedings of IEEE Congress on Evolutionary Computation (CEC 2004), pp. 486-493. IEEE Press, Los Alamitos (2004)

20. Wedge, D.C., Kell, D.B.: Rapid prediction of optimum population size in genetic programming using a novel genotype - fitness correlation. In: Proceedings of the 10th Annual Conference on Genetic and Evolutionary Computation (GECCO 2008), pp. 1315-1322. ACM, New York (2008)

21. Woolfit, M., Bromham, L.: Population size and molecular evolution on islands. Proceedings of The Royal Society B 272(1578), 2277-2282 (2005)

22. Working Group on Data Modeling Benchmarks. Created on July 20, 2002, http://neural.cs.nthu.edu.tw/jang/benchmark/

23. Yang, Z., Bielawski, J.P.: Statistical methods for detecting molecular adaptation. Trends in Ecology and Evolution 15(12), 496-503 (2000) 